\title{
An Experimental Analysis of Fuzzy C-Means and K- Means Segmentation Algorithm for Iron Detection in Brain SWI using Matlab
}

\author{
Beshiba Wilson \\ Research Scholar \\ Dept. of CSE \\ Noorul Islam University \\ Thuckalay, \\ Tamil Nadu, India
}

\author{
Julia Punitha Malar Dhas \\ Professor \& HOD \\ Dept. of CSE \\ Noorul Islam University \\ Thuckalay, \\ Tamil Nadu, India
}

\begin{abstract}
An accurate assessment of iron accumulation is required for diagnosis and therapy of iron overload in various neurodegenerative diseases. Susceptibility Weighted Imaging (SWI) offers information about any tissue that has a different susceptibility than its surrounding structures. Reliable methods to precisely quantify brain iron are essential. Image segmentation refers to partition of an image into different regions that differ in some characteristics. Accurate segmentation of medical images is a very difficult task. However, the process of accurate segmentation of these images is very important for a correct diagnosis by clinical tools. In this paper, an experimental analysis is done using fuzzy c-means and k-means segmentation algorithm for detection of iron content in SWI brain images.
\end{abstract}

\section{General Terms}

Neurodegenerative diseases, Segmentation.

\section{Keywords}

Susceptibility Weighted Imaging, Fuzzy c-means.

\section{INTRODUCTION}

Susceptibility-weighted imaging (SWI) is a neuroimaging technique, which uses tissue magnetic susceptibility differences to generate a unique contrast, different from that of spin density, T1, T2, and $\mathrm{T} 2 *$ [1]. The magnitude and phase data are combined to produce an enhanced contrast magnitude image which is sensitive to venous blood, hemorrhage and iron storage. It is well known that iron content in the brain increases with age, particularly in the basal ganglia, and that abnormal levels of iron in the central nervous system (CNS) are seen in various neurodegenerative diseases[2][3]. Susceptibility-weighted imaging of iron deposition is helpful for describing Alzheimer disease [2] and certain pediatric disorders. Iron plays an important role as a critical part of hemoglobin that transports oxygen from lung to tissues. If iron is not properly regulated, it can lead to oxidative stress and the production of free radicals any overload of ferrous iron will lead to reaction with hydrogen peroxide and the formation of aggressive hydroxyl radicals by Fenton reaction and the subsequent death of neurons by apoptosis [4]. Regions of brain like globus pallidus have high iron concentrations.

In SWI, magnitude information is combined with phase information to enhance susceptibility induced image contrast. Here we present an experimental analysis of fuzzy c-means and k-means segmentation algorithm for iron detection in brain SWI using Matlab.

\section{SEGMENTATION METHODS IN MEDICAL IMAGING}

The objective of segmentation is dividing an input image into different regions or edges with similar properties. Many methods have been developed to segment the images, many of them are based on the basic two properties namely discontinuity based and similarity based. The success of medical image analysis depends on accurate image segmentation algorithms, so an accurate segmentation of medical images is primary in clinical diagnosis and treatment planning. An overview of k-means and fuzzy c-means algorithm is provided below.

\subsection{K-Means Clustering Algorithm}

In this section, the k-means clustering algorithm $[5,6,7]$ is briefly described. The number of clusters $\mathrm{k}$ is assumed to be fixed in $\mathrm{k}$-means clustering. Let the $\mathrm{k}$ prototypes $\left(\mathrm{w}_{1}, \ldots \ldots . \mathrm{w}_{\mathrm{k}}\right)$ be initialized to one of the $n$ input patterns $\left(i_{1}, \ldots \ldots . . . i_{n}\right)$. Therefore,

$$
w_{j}=i_{l}, j \in\{1, \ldots \ldots, k\}, l \in\{1, \ldots \ldots n\}
$$

The quality of the clustering is determined by the following error function:

$$
E=\sum_{j=1}^{k} \sum_{i_{1} \in C_{j}}\left|i_{l}-w_{j}\right|^{2}
$$

$\mathrm{C}_{\mathrm{j}}$ is the $\mathrm{j}^{\text {th }}$ cluster whose value is a disjoint subset of input patterns. The appropriate choice of $\mathrm{k}$ is problem and domain dependent.

The steps of k-means clustering are as below:

Step1. Determine the number of clusters we want in the final

classified result and set the number as $\mathrm{N}$. Randomly select $\mathrm{N}$ patterns in the whole data bases as the $\mathrm{N}$ centroids of $\mathrm{N}$ clusters.

Step2. Classify each pattern to the closest cluster centroid. The closest usually represent the pixel value is similarity, but it still can consider other features.

Step3. Recompute the cluster centroids and then there have N centroids of N clusters as we do after Step1. 
Step4. Repeat the iteration of Step 2 to 3 until a convergence criterion is met. The typical convergence criteria are: no reassignment of any pattern from one cluster to another, or the minimal decrease in squared error.

\subsection{Fuzzy C-Means Algorithm}

The Fuzzy C-means is an unsupervised clustering algorithm whish can be applied to several problems involving feature analysis, clustering, medical diagnosis and image segmentation [8][9][10]. Fuzzy C-means clustering (FCM) algorithm was proposed by Bezdek et.al in which each data point belongs to a cluster to a degree specified by a membership grade.

The FCM algorithm minimizes the objective function for the partition of data set, $x=\left[\mathrm{x}_{1}, \mathrm{x}_{2}, \ldots, \mathrm{x}_{\mathrm{d}}\right]^{\mathrm{T}}$, given by: [11]

$$
J_{m}(u, v)=\sum_{\mathrm{i}=1}^{\mathrm{c}} \sum_{\mathrm{k}=1}^{\mathrm{d}} \mathrm{u}_{\mathrm{i}, \mathrm{k}}^{\mathrm{m}}\left\|\mathrm{x}_{\mathrm{k}}-\mathrm{v}_{\mathrm{i}}\right\|^{2}
$$

In the above equation, $\mathrm{d}$ is the number of samples in vector $\mathrm{X}$, $\mathrm{c}$ is the number of clusters, $\mathrm{u}_{\mathrm{ik}}$ is the element of the partition matrix $U$ of size (c $x d$ ) containing the membership function, $\mathrm{v}_{\mathrm{i}}$ is the center of the center of the $i^{\text {th }}$ class, and $\mathrm{m}$ is a weighting factor that controls the fuzziness of the membership function. The fuzzy c-means algorithm performs the partition of the vector $X$ into $c$ fuzzy subsets where $u_{i k}$ represents the membership of $x_{k}$ in class $i$.

The FCM clustering technique can be summarized by the following steps [11]:

Step 1: Initialization

- Scan the image line by line to construct the vector $\mathrm{X}$ containing all the gray level of the image

- Randomly initialize the centers of the classes vector $\mathrm{V}^{(0)}$

From the iteration $\mathrm{t}=1$ to the end of the algorithm:

Step 2: Calculate the membership matrix $\mathrm{U}^{(\mathrm{t})}$ of element $\mathrm{u}_{\mathrm{ik}}$ using:

$$
u_{i k}=\left(\sum_{j=1}^{c}\left(\frac{\left\|x_{k}-v_{i}\right\|}{\left\|x_{k}-v_{j}\right\|}\right)^{\frac{2}{m-1}}\right)^{-1}
$$

Step 3: Calculate the vector $\mathrm{V}^{(\mathrm{t})}=\left[\mathrm{v}_{1}, \mathrm{v}_{2}, \ldots, \mathrm{v}_{\mathrm{c}}\right]$ using:

$$
v_{i}=\frac{\sum_{k-1}^{d} u_{i k}^{m}}{\sum_{k-1}^{d} u_{i k}^{m}}
$$

Step 4: Convergence test: if $\left\|\mathrm{V}^{(\mathrm{t})}-\mathrm{V}^{(\mathrm{t}-1)}\right\|>\varepsilon$, then increment the iteration $\mathrm{t}$, and return to the Step 2, otherwise, stop the algorithm. $\varepsilon$ is a chosen positive threshold.

\section{IMPLEMENTATION OF K-MEANS AND FUZZY C-MEANS IN MATLAB}

The matlab function used for $\mathrm{k}$-means clustering is IDX = kmeans $(X, k)$, partitions the points in the $n$-by-p data matrix $X$ into $\mathrm{k}$ clusters. This iterative partitioning minimizes the sum, over all clusters, of the within-cluster sums of point-tocluster-centroid distances. Rows of $\mathrm{X}$ correspond to points, columns correspond to variables. kmeans returns an n-by-1 vector IDX containing the cluster indices of each point. By default, kmeans uses squared Euclidean distances.

The matlab function used for fcm(data, cluster_n) applies the fuzzy c-means clustering method to a given data set. The input arguments of this function are
- data: data set to be clustered; each row is a sample data point

- $\quad$ cluster_n: number of clusters (greater than one)

The output arguments of this function are

- center: matrix of final cluster centers where each row provides the center coordinates

- U: final fuzzy partition matrix (or membership function matrix)

- obj_fcn: values of the objective function during iterations

\section{EXPERIMENTAL RESULTS}

The K-means and FCM segmentation techniques have been implemented using Matlab 7.9.0 (R2009b). The performance of various SWI brain images is analyzed. The RMSE and PSNR are used to evaluate the segmentation of medical image. Table 1 provides the PSNR and RMSE values for different SWI images. The extraction of the iron region in brain is made by k-means and Fuzzy C--means clustering method. The SWI is compared for brain iron using k-means and FCM methods.

Table 1: PSNR and RMSE values for different SWI images

\begin{tabular}{|c|c|c|c|c|}
\hline \multirow{2}{*}{ S.No } & \multicolumn{2}{|c|}{ K-Means } & \multicolumn{2}{c|}{ FCM } \\
\cline { 2 - 5 } & PSNR & RMSE & PSNR & RMSE \\
\hline 1 & 23.1789 & 105.209 & 33.6745 & 112.5871 \\
\hline 2 & 25.6634 & 108.241 & 36.1041 & 115.2132 \\
\hline 3 & 26.3142 & 121.258 & 38.1659 & 119.2143 \\
\hline
\end{tabular}

The input image is the SWI brain image for detecting iron content. The tests done on fuzzy c-means indicates that the iron regions are easily visible than the output of $\mathrm{k}$-means image. Fig 1. shows the k-means and fuzzy c-means segmented brain SWI images for detection of iron content.

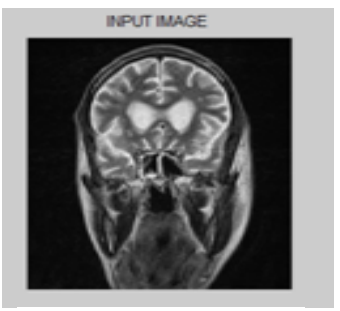

Fig 1(A): Input SWI brain image

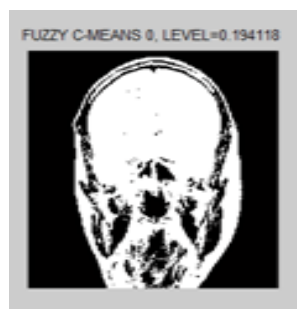

Fig 1(B) FCM segmented image

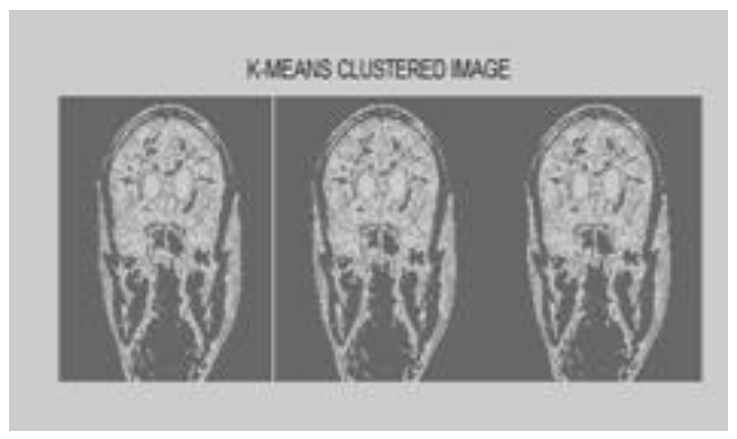

Fig 1(C) K-Means segmented image brain 


\section{CONCLUSION}

In this paper implementation of k-means and fuzzy c-means segmentation algorithm for detecting iron region in brain was successfully done using Matlab. This helps in the clinical diagnosis of brain iron in SWI images. This decision can assist as a supportive aid which can be used at the doctor's discretion in finally declaring a decision.

The accurate results of Fuzzy C-means clustering algorithm effectively extract the iron content from brain SWI images. The Fuzzy C--means algorithm is used because of its simplicity and its clustering rate is faster than the other method. This work was effective in extracting the iron content in brain and this can be extended to abnormality detection in brain.

\section{REFERENCES}

[1] E.M. Haacke, S. Mittal, Z. Wu, J. Neelavalli, Y.-C.N. Cheng, Susceptibility-Weighted Imaging: Technical Aspects and Clinical Applications, Part 1, American Journal of Neuroradiology, 30:19 -30, Jan 2009.

[2] E.M. Haacke , Cheng N Y, House M J, et al., Imaging Iron Stores In The Brain Using Magnetic Resonance Imaging, Magn Reson Imaging 2005,23:1-25.

[3] Harder S L, Hopp K M, Ward H, et al. , Mineralization of the deep gray matter with age: a retrospective review with susceptibility-weighted MR imaging, American Journal of Neuroradiology ,2008, 29:176 -83.
[4] Gelman B B, Iron in CNS disease, J Neuropathol Exp Neurol, 1995, 54:477- 86.

[5] R. C. Dubes and A. K. Jain. Algorithms for Clustering Data, Prentice Hall, 1988.

[6] L. Kaufman and P. J. Rousseeuw. Finding Groups in Data: an Introduction to Cluster Analysis. John Wiley \& Sons, 1990.

[7] K. Mehrotra, C. Mohan, and S. Ranka. Elements of Artificial Neural Networks. MIT Press, 1996.

[8] L. O. Hall, A. M. Bensaid, L. Clarke, R. P. Velthuizen, M. Silbiger, and J.C. Bezdek, A comparison of neural network and fuzzy clustering techniques in segmenting magnetic resonance images of the brain, IEEE Trans. Neural Networks, 3 (1992), 672 - 681.

[9] R.M. Haralick, K. Shanmugam and I. Dinstein, Textural features for image classification, IEEE Trans. Syst. Man and Cyber.,3 (1973), 610 - 621.

[10] W. Chumsamrong, P. Thitimajshima, and Y. Rangsanseri, Synthetic aperture radar (SAR) image segmentation using a new modified fuzzy c-means algorithm, Proceedings of Geoscience and Remote Sensing Symposium, 2 (2000), 624 - 626.

[11] J.C. Bezdek, Pattern recognition with fuzzy objective function algorithms, Plenum, New York, 1981 